\title{
Structure-Activity Relationship Analysis of Psychedelics in a Rat Model of Asthma Reveals the Anti-Inflammatory Pharmacophore
}

Thomas W. Flanagan ${ }^{1}$, Gerald B. Billac ${ }^{1}$, Alexus N. Landry ${ }^{1}$, Melaine N. Sebastian ${ }^{1}$, Stephania A. Cormier ${ }^{2}$, and ${ }^{*}$ Charles D. Nichols ${ }^{1}$

1. Department of Pharmacology and Experimental Therapeutics Louisiana State University Health Sciences Center New Orleans, LA 70112

2. Department of Biological Sciences Louisiana State University 202 Life Sciences Building Baton Rouge, LA 70803 


\section{Supporting Information}

\section{$\underline{\text { Table of Contents }}$}

-Supplementary Figure

Figure S1. (R)-DOI fails to prevent OVA-induced AHR in HTR2A-/- knockout mice.

-Supplementary Materials and Methods

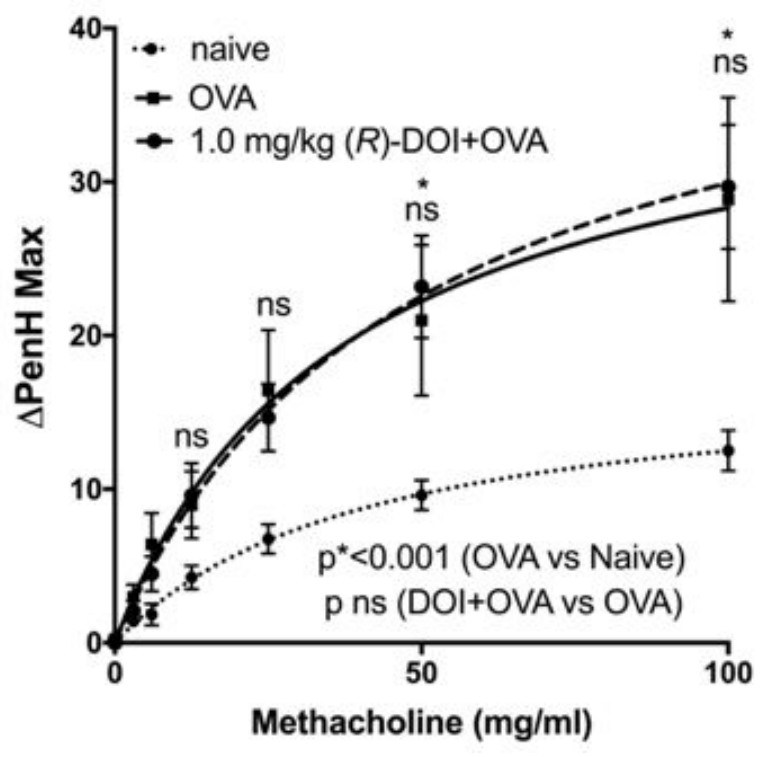


Figure S1. (R)-DOI fails to prevent OVA-induced AHR in HTR2A-knockout mice. Results

from whole body plethysmography on awake, freely moving $5-\mathrm{HT}_{2 \mathrm{~A}}$ receptor knockout mice exposed to $0.5 \mathrm{mg} / \mathrm{kg}(R)$-DOI (inhaled). $\mathrm{n}=7-8$ mice/treatment group; ${ }^{*} \mathrm{p}<0.001$ OVA vs Naïve; ns = no significance Drug vs OVA. Error bars represent \pm SEM; 2-way ANOVA with Bonferonni

\section{Supplemental Materials and Methods}

\section{Animals}

Generation of null-mutation mice lacking $5-\mathrm{HT}_{2 \mathrm{~A}}$ receptor expression was previously described ${ }^{54}$. Breeder HTR2A ${ }^{-/-}$mice in a $129 \mathrm{~Sv}$ background (129v5HT2a-/-) 55 were generously provided by Dr. Javier González-Maeso (Virginia Commonwealth University School of Medicine, Richmond, VA). Animal care and experimental procedures were approved by the Institutional Animal Care and Use Committee at Louisiana State University Health Sciences Center. All animals were maintained in the animal care facility at the Louisiana State University Health Sciences Center (New Orleans, LA) in a pathogen-free environment with ad libitum access to food and water on a $12 \mathrm{hr}$ light/dark cycle (lights out at 7:00 P.M.). 
Twenty-four male 6-8 wk old male mice weighing between 20-25 gm were sensitized and challenged with chicken OVA Grade V (Sigma-Aldrich), as previously described ${ }^{11}$. Sensitization occurred via intraperitoneal injection (100 $\left.\mu \mathrm{l}\right)$ of $20 \mu \mathrm{g}$ OVA emulsified in $2 \mathrm{~mL}$ Imject Alum $\left[\mathrm{Al}(\mathrm{OH})_{3} / \mathrm{Mg}(\mathrm{OH})_{2}\right.$; Pierce] on days 0 and 7 . On days 1416 OVA and DOI+OVA mice were placed in a 7 liter $(21 \times 22 \times 15 \mathrm{~cm})$ clear plexiglass induction chamber (Vet Equip, Inc., Pleasanton, CA) and challenged for 20 min with a 1\% (wt/vol) OVA solution in sterile saline (Baxter Healthcare Corp.) generated using a VixOne reusable nebulizer (Westmed) powered by a PARI PRONEB Ultra compressor (Pari Proneb). Naïve animals were challenged as above with sterile saline. Thirty min. prior to saline/OVA challenge, each animal was placed in a nylon-coated stainless-steel wire

restraint (SoftRestraints;SciReq, Montreal, PQ, Canada) and treated with 1.0 $\mathrm{mg} / \mathrm{kg}(R)$-DOI or saline control (nose-only inhalation) once daily using an ultrasonic nebulizer (Aeroneb Pro).

\section{Non-invasive whole-body plethysmography and methacholine challenge}

$48 \mathrm{~h}$ post final OVA challenge airway function was measured in a single chamber, WBP system (Buxco Electronics and EMKA Technologies) in unrestrained, conscious mice. PenH was used as a proxy for AHR as previously described ${ }^{42}$. For our studies all animals were placed in a single chamber for a 10 min acclimatization period; animals were subsequently measured for baseline PenH 3 min prior to challenge. Mice were exposed for $1 \mathrm{~min}$ to the bronchoconstrictor MeCh (Sigma-Aldrich) at increasing concentrations $(0,3.125,6.25,12.5,50$, and $100 \mathrm{mg} / \mathrm{mL}$ in isotonic saline) and PenH was recorded for $3 \mathrm{~min}$. The maximal PenH was analyzed using the Datanlyst software 
(Datanalyst v2.6.1.14), averaged for each dose, and plotted as percent change from vehicle controls, as described ${ }^{12}$.

\section{Statistics}

All statistical analysis was performed using GraphPad Prism (GraphPad Software, La Jolla, CA). 